\title{
Antiproton modulation in the Heliosphere and AMS-02 antiproton over proton ratio prediction
}

\author{
P. Bobik ${ }^{1}$, M. J. Boschini ${ }^{2,4}$, C. Consolandi ${ }^{2}$, S. Della Torre ${ }^{2,5}$, M. Gervasi ${ }^{2,3}$, D. Grandi ${ }^{2}$, K. Kudela ${ }^{1}$, S. Pensotti ${ }^{2,3}$, \\ and P. G. Rancoita ${ }^{2}$ \\ ${ }^{1}$ Institute of Experimental Physics, Kosice, Slovak Republic \\ ${ }^{2}$ Istituto Nazionale di Fisica Nucleare, INFN Milano-Bicocca, Milano, Italy \\ ${ }^{3}$ Department of Physics, University of Milano Bicocca, Milano, Italy \\ ${ }^{4}$ CILEA, Segrate (MI), Italy \\ ${ }^{5}$ Department of Physics and Maths, University of Insubria, Como, Italy
}

Received: 15 November 2010 - Revised: 22 January 2011 - Accepted: 30 January 2011 - Published: 4 July 2011

\begin{abstract}
We implemented a quasi time-dependent 2-D stochastic model of solar modulation describing the transport of cosmic rays (CR) in the heliosphere. Our code can modulate the Local Interstellar Spectrum (LIS) of a generic charged particle (light cosmic ions and electrons), calculating the spectrum at 1 AU. Several measurements of CR antiparticles have been performed. Here we focused our attention on the $\mathrm{CR}$ antiproton component and the antiproton over proton ratio. We show that our model, using the same heliospheric parameters for both particles, fit the observed $\bar{p} / p$ ratio. We show a good agreement with BESS-97 and PAMELA data and make a prediction for the AMS-02 experiment.
\end{abstract}

\section{Introduction}

Galactic cosmic rays (GCRs) are nuclei, with a small component of leptons, mainly produced by supernova remnants (Blasi, 2010), confined by the galactic magnetic field to form a isotropic flux inside the galaxy. Before reaching the Earth orbit they enter the heliosphere, the region where the interplanetary magnetic field is carried out by the solar wind (SW). In this enviroment they undergo diffusion, convection, magnetic drift and adiabatic energy loss, resulting in a reduction of particles flux at low energy $(\leq 1-10 \mathrm{GeV})$ depending on solar activity and polarity. This effect is known as solar modulation. We have developed a 2-D (radius and heliocolatitude) model of GCR propagation (Bobik et al., 2003) in the heliosphere, by using stochastic differential equations (SDEs). The model depends on measured values of the SW velocity on the ecliptic plane $\left(V_{0}\right)$, tilt angle $(\alpha)$ of the neutral

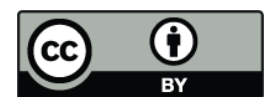

Correspondence to: D. Grandi (davide.grandi@mib.infn.it) sheet and estimated values of the diffusion parameter $\left(k_{0}\right)$ : details on parameters are discussed in Sects. 2 and 3. This model includes drift transport due to magnetic field curvature and gradients, as well the presence of a tilted neutral sheet describing properly periods of low and medium solar activity. Modulated fluxes depend on solar activity but also on particle charge and solar magnetic polarity (Boella et al., 2001).

\section{Stochastic 2-D Monte Carlo code}

The GCR transport in the Heliosphere is described by a Fokker-Planck equation, the so-called Parker equation (Parker, 1965):

$$
\begin{aligned}
\frac{\partial U}{\partial t}= & \frac{\partial}{\partial x_{i}}\left(K_{i j}^{S} \frac{\partial U}{\partial x_{j}}\right)-\frac{\partial}{\partial x_{i}}\left(V_{s w_{i}} U\right) \\
& +\frac{1}{3} \frac{\partial V_{s w_{i}}}{\partial x_{i}} \frac{\partial}{\partial T}(\varrho T U)-\frac{\partial}{\partial x_{i}}\left(v_{D_{i}} U\right)
\end{aligned}
$$

where $U$ is the cosmic ray number density per unit interval of particle kinetic energy, $t$ is the time, $T$ is the kinetic energy (per nucleon), $V_{s w_{i}}$ the SW velocity along the axis $x_{i}$, $v_{D_{i}}$ is the drift velocity related to the antisymmetric part of diffusion tensor (Jokipii and Levy, 1977), $K_{i j}^{S}$ is the symmetric part of the diffusion tensor and $\varrho=\left(T+2 T_{0}\right) /\left(T+T_{0}\right)$ (Gleeson and Axford, 1967), where $T_{0}$ is particle's rest energy. This partial differential equation is equivalent (Gardiner, 1989) to a set of ordinary SDEs that can be integrated with Monte Carlo (MC) techniques. The integration time step $(\Delta t)$, is taken to be proportional to $r^{2}$ ( $r$ is the distance from the Sun) avoiding oversampling in the outer heliopshere and therefore saving CPU time (Alanko-Huotari et al., 2007). We considered the 2-D (radius and colatitude) approximation

Published by Copernicus Publications on behalf of the Arbeitsgemeinschaft Extraterrestrische Forschung e.V. 


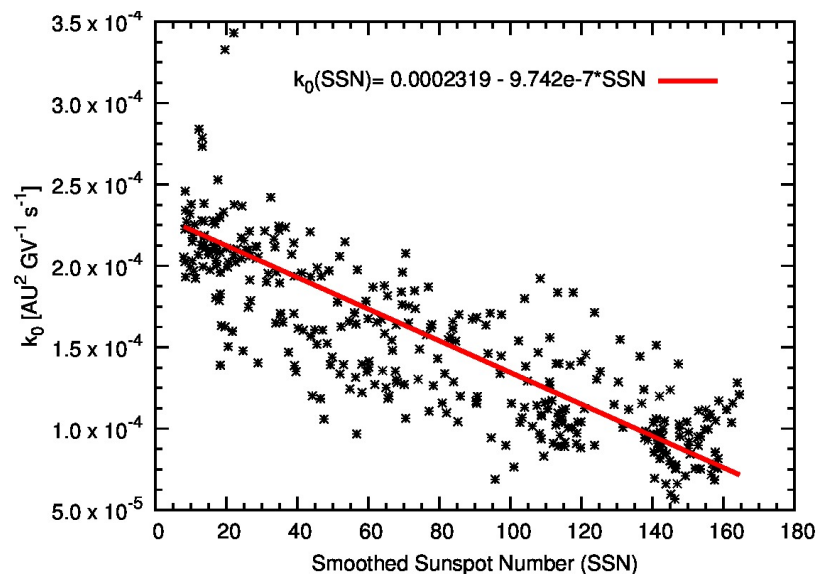

Fig. 1. $k_{0}$ values versus monthly SSN. The linear fit is also shown. Reported values cover the time period 1951-2004.

of Eq. (1), and from this we calculate the equivalent set of SDEs:

$$
\begin{aligned}
\Delta r= & \frac{1}{r^{2}} \frac{\partial\left(r^{2} K_{r r}\right)}{\partial r} \Delta t+\left(V_{s w}+v_{D_{r}}+v_{D_{N S}}\right) \Delta t \\
& +R_{g} \sqrt{2 K_{r r} \Delta t} \\
\Delta \mu= & \frac{1}{r^{2}} \frac{\partial\left[\left(1-\mu^{2}\right) K_{\theta \theta}\right]}{\partial \mu} \Delta t-\left(\frac{\sqrt{1-\mu^{2}}}{r} v_{D_{\theta}}\right) \Delta t \\
& +R_{g} \sqrt{\frac{2 K_{\theta \theta}\left(1-\mu^{2}\right)}{r^{2}} \Delta t} \\
\Delta T= & -\left(\frac{2}{3} \frac{\varrho V_{s w} T}{r}\right) \Delta t
\end{aligned}
$$

where $\mu=\cos \theta$, with $\theta$ colatitude, and $R_{g}$ is a gaussian distributed random number with unitary variance. Here the drift velocity is split in regular drift (radial drift $v_{D_{r}}$, latitudinal drift $v_{D_{\theta}}$ ) and neutral sheet drift $\left(v_{D_{N S}}\right)$ as described by Hattingh and Burger (1995). The radial diffusion coefficient is $K_{r r}=K_{\|} \cos ^{2} \psi+K_{\perp r} \sin ^{2} \psi$ (Potgieter et al., 1993), where $\psi$ is the angle between radial versor and direction of the solar magnetic field described below. The latitudinal coefficient is $K_{\theta \theta}=K_{\perp \theta}$ (e.g. see Potgieter and Le Roux, 1994). We note as the perpendicular diffusion coefficient has two components, one in the radial direction $\left(K_{\perp r}\right)$ and one in the polar direction $\left(K_{\perp \theta}\right)$ as shown in Potgieter (2000). We define $\left(K_{\perp}\right)_{0_{i}}$ as the ratio between perpendicular and parallel diffusion coefficients, therefore $K_{\perp i}=\left(K_{\perp}\right)_{0_{i}} K_{\|}$. We fixed this value: $\left(K_{\perp}\right)_{0_{r}}=0.05$ while $\left(K_{\perp}\right)_{0_{\theta}}=f(\theta)\left(K_{\perp}\right)_{0_{r}}$ (where $f(\theta)=10$ close to the poles and $f(\theta)=1$ in the equatorial region) (Potgieter, 2000), to reproduce the correct magnitude and rigidity dependence of the latitudinal cosmic ray proton and electron gradients (cf. Potgieter, 1997; Burger et al., 2000). The parallel diffusion coefficient is $K_{||}=k_{0} \beta K_{P}(P)\left(B_{\oplus} / 3 B\right)$ (Potgieter and Le Roux, 1994); here $k_{0} \simeq 0.05-0.3 \times 10^{-3} \mathrm{AU}^{2} \mathrm{GV}^{-1} \mathrm{~s}^{-1}$, is a diffusion parameter depending on the solar actvitiy (see Sect. 3 ), $\beta$ is the particle velocity, $P$ is the CR particle's rigidity, $K_{P}=P, B_{\oplus}$ is the value of heliospheric magnetic field at the Earth orbit, and $B$ is the magnitude of the Heliospheric Magnetic Field (HMF) (Hattingh and Burger, 1995):

$\mathbf{B}=\frac{A}{r^{2}}\left(\mathbf{e}_{r}-\Gamma \mathbf{e}_{\phi}\right)\left[1-2 H\left(\theta-\theta^{\prime}\right)\right]$

where $A$ is a coefficient that determines the field polarity and allows $|\mathbf{B}|$ to be equal to $B_{\oplus}$, i.e. the value of IMF at the Earth orbit; $\theta^{\prime}$ is the polar angle determining the position of the heliospheric current sheet (HCS) (Jokipii and Thomas, 1981); $H$ is the Heaviside function, thus $\left[1-2 H\left(\theta-\theta^{\prime}\right)\right]$ for the change of sign between the two regions - above and below the HCS - of the heliosphere; finally $\Gamma=\tan \psi \simeq \omega r \sin \theta / V_{s w}$, with $\psi$ the spiral angle. The Parker field has been modified introducing a small latitudinal component $B_{\theta}=\frac{A}{r^{2}}\left(r / r_{0}\right) \delta(\theta)$ with $\delta(\theta)=8.7 \times 10^{-5} / \sin \theta$, thus allowing one to obtain $\nabla \cdot \mathbf{B}=0$ and a field magnitude according to Jokipii and Kóta (1989):

$B=\frac{A}{r^{2}} \sqrt{1+\Gamma^{2}+\left(\frac{r}{r_{0}}\right)^{2} \delta^{2}}$

that increases the magnitude of the HMF in the polar regions without a modification of the field topology. This component produces a lower magnetic drift velocity in this region, with the effect of a lower CR penetration along polar field lines in the inner part of the heliosphere (Jokipii and Kóta, 1989). We use a SW broad smoothed profile according to Ulysses data for periods of low solar activity (Mc Comas et al., 2000), described by the relation $V_{s w}(\theta)=V_{\max }$ if $\theta \leq 30^{\circ}$ or $\theta \geq 150^{\circ}$ and $V_{s w}(\theta)=V_{0} \cdot(1+|\cos \theta|)$ if $30^{\circ}<\theta<150^{\circ}$ where $V_{0}$ is approximately $400 \mathrm{~km} / \mathrm{s}$ and $V_{\max }$ is $760 \mathrm{~km} / \mathrm{s}$. Drift effects are included through analytical effective drift velocities: in the Parker spiral field we evaluated drift due to gradient, curvature and neutral sheet that modify the integration path inside the heliosphere. We adopted the approach of Potgieter and Moraal (1985), because it is able to reproduce the effects of drift in both quiet and active solar periods (a discussion on other models can be found in Bobik et al., 2010). In this model the drift coefficient is modified with a transition function that simulates the effect of a wavy neutral sheet. The sharpness of this function is related to $\alpha$ angle, expanding or shrinking the region of influence of neutral sheet drift. As LIS, both for protons and antiprotons, we use the ones used in Casaus (2009) and obtained from Galprop ${ }^{1}$.

\section{Parameters and Data Sets}

Values of the tilt angle $\alpha$ are computed using two different models, described in Hoeksema (1995), fitting separately periods of increasing solar activity and periods of decreasing

\footnotetext{
${ }^{1}$ http://galprop.stanford.edu/webrun/
} 


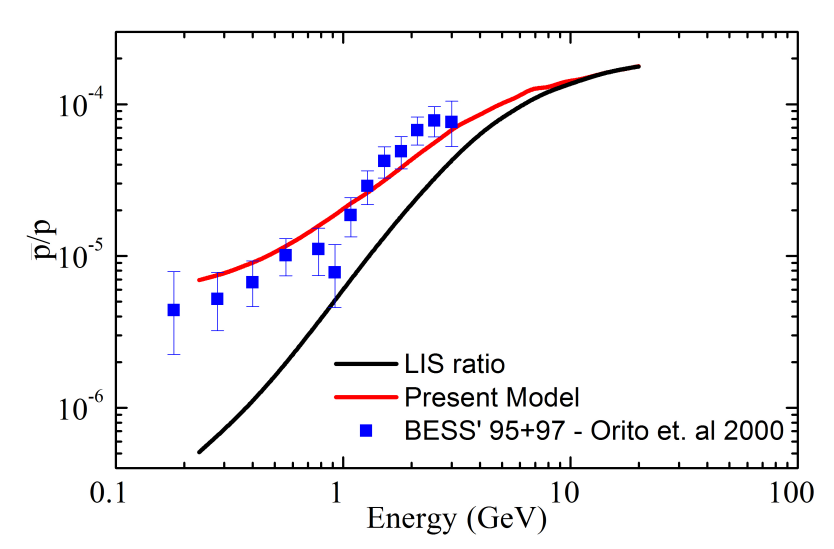

Fig. 2. Comparison of simulated $\bar{p} / p$ ratio at $1 \mathrm{AU}$ and experimental data: BESS (1997).

solar activity (Ferreira and Potgieter, 2004). The three drift components do not depend on external parameters, except the solar polarity, so $A>0$ for positive periods and $A<0$ for negative periods (Bobik et al., 2003). We selected CR $p$ and $\bar{p}$ data from several experiments in order to compare and tune model results. We modulated separately $p$ and $\bar{p}$ LIS spectra and then we computed the ratio. In this paper we show experimental data taken during periods of low solar activity: the comparison with BESS-97 ( $A>0$ July 1997 , see Orito et al., 2000) and PAMELA $(A<0$ from 2007 to 2008, see Adriani et al., 2010). $V_{0}$ and $B_{\oplus}$ values for these periods were obtained from NSSDC OMNIWeb system ${ }^{2}$ by 27 daily averages, while tilt angle values from the Wilcox Solar Laboratory (Hoeksema, 1995). We estimated the values of $k_{0}$, needed to evaluate the CR modulation in different conditions, from the modulation parameter reported in Usoskin et al. (2005). We searched a relation between the estimated $k_{0}$ values and the monthly Smoothed Sunspot Numbers (SSN). We found that there is a nearly linear relation between $k_{0}$ and $\mathrm{SSN}^{3}$ values (see Fig. 1), with a Gaussian distribution of the best fit with a RMS of $19 \%$. This is a first crude estimation, we will perform a more complex anaysis, e.g. fitting separately different solar phases, in order to avoid systematics in the relation and to reduce the RMS. In this way we can use the estimated SSN values to obtain the diffusion coefficient $k_{0}$. Following this approach we introduced in our code a gaussian random variation of $k_{0}$ with a RMS of $19 \%$. Results of the simulation with and without the gaussian variation are consistent inside the indetermination of the code (around 5\%). Our code simulates a diffusive propagation of a CR entering the heliosphere from its outer limit, that we located at $100 \mathrm{AU}$ (note that in Decker et al. (2005) the Termination Shock is located at $94 \mathrm{AU}$ ), and reaching the Earth at $1 \mathrm{AU}$; the effects of heliosheath and termination shock are

\footnotetext{
2 http://omniweb.gsfc.nasa.gov/form/dx1.html

${ }^{3} \mathrm{http} / / / \mathrm{www}$. sidc.oma.be/sunspot-data/
}

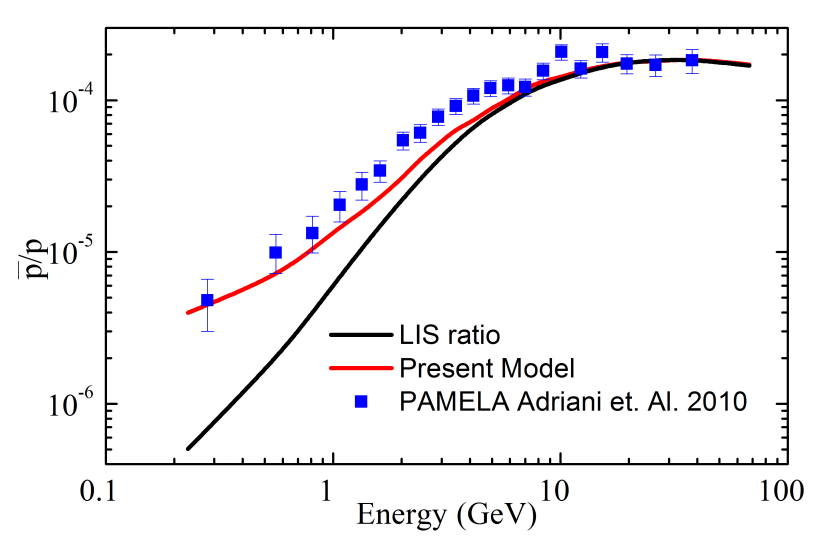

Fig. 3. Comparison of simulated $\bar{p} / p$ ratio at $1 \mathrm{AU}$ and experimental data: PAMELA (2007-2008).

not taken into account in the present model. We evaluated the time $t_{s w}$ needed by the SW to expand from the outer corona up to $100 \mathrm{AU}$, with a minimum speed of $\sim 400 \mathrm{~km} / \mathrm{s}$ it takes nearly 14 months, while the time interval $\tau_{e v}$ of the stochastic evolution of a quasi particle inside the heliosphere from $100 \mathrm{AU}$ down to $1 \mathrm{AU}$ is between 1 month (at $200 \mathrm{MeV}$ ) and few days (at $10 \mathrm{GeV}$ ). This scenario, where $\tau_{e v}<t_{s w}$ and $t_{s w} \gg 1$ month, indicates that we should use different parameters (monthly averages) to describe the conditions of heliosphere in the modulation process. In fact at $100 \mathrm{AU}$, where particles are injected, the conditions of the solar activity are similar to those present at the Earth $\sim 14$ months before. Therefore, we can divide the heliosphere in 14 regions as a function of the radius. For each region we evaluated $k_{0}, \alpha$ and $V_{s w}$, in relation to the time spent by the solar wind to reach this region. We indicate the present treatment accounting for the time evolution of the solar parameters as a dynamic approach of the heliosphere.

\section{Results}

Results obtained with our propagation code are shown in Figs. 2 and 3. Simulated fluxes obtained using parameters dependent on the heliospheric region agree with measured data within the experimental error bars. This happens both in periods with $A>0$ (BESS-97), and in periods with $A<0$ (PAMELA). This means that current treatment of the Heliosphere improves the understanding of the complex processes occurring inside the Solar Cavity. Our code can be also used to predict CR fluxes for future measurements. The assumption is that diffusion coefficient, tilt angle and solar wind speed show a near-regular and almost periodic trend. The periodicity is two consecutive 11-years solar cycles. We selected periods with a similar solar activity conditions and same solar field polarity of the time of interest: therefore approximately 22 years before. We used the values measured in 


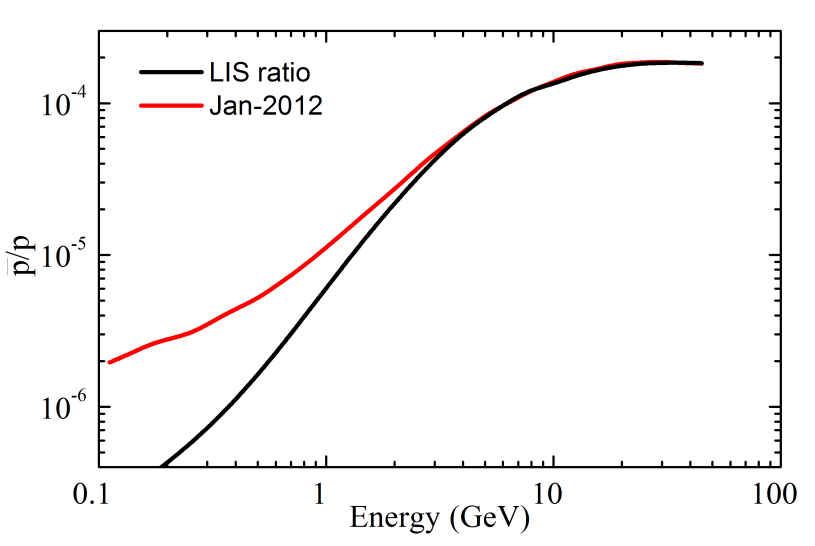

Fig. 4. Prediction of modulated $\bar{p} / p$ ratio at 1 AU for AMS-02.

that periods as an estimation of the conditions of the heliosphere. Simulations have been carried out in prevision of the AMS-02 mission that will be installed on the ISS in 2011: we choose January 2012. For this period we show in Fig. 4 the predictions of GCR modulation for the $\bar{p} / p$ ratio. In order to reduce the uncertainty it is important to compare our model with the AMS-02 data because of the huge statistics and the long time covered.

\section{Conclusions}

We developed a 2-D stochastic MC code for particles propagation across the heliosphere. We compared the ratios of $\bar{p} / p$ fluxes measured by BESS and PAMELA with those obtained from the present $\mathrm{MC}$ code. In the present calculations we used - for the parameters $k_{0}, \alpha$ and $V_{s w}$ - values corresponding to the periods of data taking. This description of the heliosphere and the forward approach seem to properly account for the propagation of GCR in the solar cavity. Recent measurements (Adriani et al., 2010) have pointed out the needs to reach a high level of accuracy in the modulation of the fluxes, in relation to the charge sign of the particles and the solar field polarity. This aspect will be even more crucial in the next generation of experiments like AMS-02.

Edited by: B. Heber

Reviewed by: A. Kopp and another anonymous referee

\section{References}

Adriani, O., Barbarino, G. C., Bazilevskaya, G. A., et al.: PAMELA results on the cosmic-ray antiproton flux from $60 \mathrm{MeV}$ to $180 \mathrm{GeV}$ in kinetic energy, Phys. Rev. Lett., 105, 121101, 2010.

Alanko-Huotari, K., Usoskin, I. G., Mursula, K. and Kovaltsov, G. A.: Stochastic simulation of cosmic ray modulation including a wavy heliospheric current sheet, J. Geophys. Res., 112, A08101, 2007.
Blasi, P.: Cosmic Ray Acceleration in Supernova Remnants, 1th CRICATPP Conference 2010.

Bobik, P., Boschini, M., Gervasi, M., et al.: Cosmic ray spectrum at $1 \mathrm{AU}$ : a transmission function approach to the magnetosphere, in: Proc. ICSC 2003, edited by: A. Wilson, ESA SP-535, ISBN 92-9092-845-X, 2003, 637-640, 2003.

Bobik, P., Boella, G., Boschini, M. J., et al.: Drift Models and Polar Field for Cosmic Rays Propagation in the Heliosphere, in: Proc. of 11th ICATPP Conf., Como, Italy, 5-9/10/2009, 760, DOI: 10.1142/9789814307529_0121, 2010.

Boella, G., Gervasi, M., Potenza, M. A. C., et al.: Modulated antiproton fluxes for interstellar production models, Astropart. Phys., 9, 261, 1998.

Boella, G., Gervasi, M., Mariani, S., et al.: Evidence for charge drift modulation at intermediate solar activity from the flux variation of protons and $\alpha$ particles, J. Geophys. Res., 106, 29355, 2001.

Burger, R. A., Potgieter, M. S., and Heber, B.: Rigidity dependence of cosmic ray proton latitudinal gradients measured by the Ulysses spacecraft: Implications for the diffusion tensor, J. Geophys. Res., 105, 27447-27456, 2000.

Casaus, J.: The AMS-02 experiment on the ISS, J. Phys. Conf. Ser., 171, 012045, 2009.

Decker, R. B., Krimigis, S. M., Roelof, E. C., et al: Voyager 1 in the Foreshock, Termination Shock, and Heliosheath Science, 309, 2020-2024, 2005.

Ferreira, S. E. S. and Potgieter, M. S.: Long-Term Cosmic-Ray Modulation in the Heliosphere, Astrophys. J., 603, 744, 2004.

Gardiner, C. W.: Handbook of Stochastic Methods, Springer Verlag, Berlin, 1989.

Gleeson, L. J. and Axford, W. I.: Cosmic Rays in the Interplanetary Medium, Astrophys. J., 149, 115, 1967.

Gleeson, L. J. and Axford, W. I.: Solar Modulation of Galactic Cosmic Rays, Astrophys. J., 154, 1011, 1968.

Hattingh, M. and Burger, R. A.: A new simulated wavy neutral sheet drift model, Adv. Space Res., 16, 213, 1995.

Hoeksema, J. T.: The Large-Scale Structure of the Heliospheric Current Sheet During the ULYSSES Epoch, Space Science Rev., 72, 137-148, 1995.

Jokipii, J. R. and Levy, E. H.: Effects of particle drifts on the solar modulation of galactic cosmic rays. II, Astrophys. J., 213, 85-88, 1977.

Jokipii, J. R. and Thomas, B.: Effects of drift on the transport of cosmic rays IV. Modulation by a wavy interplanetary current sheet, Astrophys. J., 243, 1115-1122, 1981.

Jokipii, J. R. and Kóta, J.: The polar heliospheric magnetic field Geophys. Res. Lett., 16, 1989.

Mc Comas, D. J., Barraclough, B. L., Funsten, H. O., et al.: Solar wind observation over ulysses' first full polar orbit, J. Geophys. Res., 105, 10419-10434, 2000.

Orito, S., Maeno, T., Matsunaga, H., et al.: Precision Measurement of Cosmic-Ray Antiproton Spectrum, Phys. Rev. Lett., 84, 10781081, 2000.

Parker, E. N.: The passage of energetic charged particles through interplanetary space, Planet. Space Sci., 13, 9, 1965.

Potgieter, M. S., Alanko-Huotari, K., Kovaltsov, G. A., Mursula, K.: Implications of Enhanched Perpendicular Diffusion in the Heliospheric Modulation of Cosmic Rays, in: Proc. the 25th ICRC, 1997. 
Potgieter, M. S.: Heliospheric modulation of cosmic ray protons: Role of enhanced perpendicular diffusion during periods of minimum solar modulation, J. Geophys. Res., 105, 18295-18304, 2000.

Potgieter, M. S. and Moraal, H.: A drift model for the modulation of galactic cosmic rays, Astrophys. J., 294, 425-440, 1985.

Potgieter, M. S. and Le Roux, J. A.: The Long-Term Heliospheric Modulation of Galactic Cosmic Rays according to a Time-dependent Drift Model with Merged Interaction Regions, Astroph. J., 423, 817, 1994.
Potgieter, M. S., Le Roux, J. A., Burlaga, L. F., McDonald, F. B.: The role of merged interaction regions and drafts in the heliospheric modulation of cosmic rays beyond $20 \mathrm{AU}$ - A computer simulation, Astroph. J., 403, 760, 1993.

Usoskin, I. G., Alanko-Huotari, K., Kovaltsov, G. A., Mursula, K. : Heliospheric modulation of cosmic rays: Monthly reconstruction for 1951-2004, J. Geophys. Res., 110, A12108, 2005. 